\title{
Tensile Tests on Reinforcing Steel Bars in the Nigerian Construction Industry
}

\author{
Ejeh, S. P. ${ }^{1}$, JIBRIN, M. U. ${ }^{2}$ \\ 1Professor Stephen Pinder Ejeh is a Professor of Civil Engineering, former Dean of Engineering and former \\ Head of Civil Engineering Department, all in Ahmadu Bello University Zaria-Nigeria. \\ ${ }^{2}$ Mohammed Umar Jibrin is a Director at the National Board for Technology Incubation (Federal Ministry of \\ Science and Technology), Abuja-Nigeria and a PhD Student in the Department of Civil Engineering , Ahmadu \\ Bello University, Zaria - Nigeria.
}

\begin{abstract}
Tensile behaviour was investigated for reinforcing steel bars used in the Nigerian Construction Industry; this was done to ascertain the level of conformity of the tested parameters with the standards. A total of thirteen (13) companies operating in Nigeria were considered and (19) nineteen samples selected randomly with each sample containing ten specimens which were used in the tests. Out of the nineteen (19) samples, thirteen (13) were locally produced in Nigeria, while six (6) were imported. Thus, a total of 190 specimens were used for the experiment. It was found that eleven (11) samples out of the nineteen (19) samples examined failed to meet the requirements of BS4449:1997 in respect of the characteristic strength. In case of the Ultimate: Yield ratio, while only one (1) out of the nineteen (19) samples did not record the minimum values of 1.25 as prescribed by the code.
\end{abstract}

Key words: Yield, Ultimate, And Tensile: Yield Ratio.

\section{Introduction}

Reinforcing steel bars play a key role as a construction material whose properties must be known to the users before being applied for design or construction purposes.

Steel reinforcing bars available in the Nigeria's Construction Industry are obtained from both internal and external sources. The internal sources come mainly from both the indigenous major plants and the mini mills located in different parts of the country. Imported steel bars coming into the country are mainly from Russia and Ukraine. Others are those procured for specific uses by multinational companies for some specific projects, and are imported directly by the multinational company concerned.

Most local construction companies in Nigeria make all their reinforcing steel procurements from the open local markets with-out any technical information that guide users on the appropriate use.

A general survey on steel at random from the accessible companies was carried out on these samples. Out of the nineteen (19) samples from where specimens were collected, one (1) sample failed completely the bend test and five (5) samples failed out of the nineteen for the elongation. Many of them are foreign samples. Thus, the need for testing reinforcing bars so as to obtain information on their properties prior to use cannot be over emphasized.

\subsection{General Survey}

\section{Samples Preparation And Tests}

A general survey of all Steel rolling mills in the country was undertaken and samples from those into production were taken for these tests.

From the survey, it was observed that the idea that led to the development of Iron and Steel Industry in Nigeria was born in 1958 by the Federal Government. That the steel rolling mills in the country can be classified in three phases or divisions as follows: (a) Ajaokuta Steel (b) Delta Steel Company with inland rolling mills at Katsina, Jos and Osogbo. Last group is (c) the private mini mills combined together. While Ajaokuta is yet to be completed, Delta's concept is to process and pass billets as raw materials for Katsina, Jos and Osogbo rolling mills. Then the mini mills which are mainly foreign dominated are privately owned with many of them being in operation before the establishment of the ones earlier stated Above Code Value. For example, the first steel plant in Nigeria was in the group of mini plants and was established at Emene near Enugu in 1962 by the Eastern Nigerian Government. These were followed by two (2) similar plants which were established in Lagos in 1968 and 1970 respectively by private initiative.

\subsection{Samples Collection}

The samples were collected from 14 different companies as earlier stated. Six of the companies are foreign and their actual names are not known but only the countries of origin are specified. Thus, there are 
nineteen samples from fourteen different companies including the foreign ones which were considered for the test.

The Sample diameters collected from the local sources depended on the available sizes as at the time of the research within the company. For samples from foreign sources, only diameters available in the Nigerian Construction Industry / market were collected.

\subsection{Samples' Labeling}

All the fourteen companies from where the samples were collected were labeled in an alphabetical order as A, B, C ...N. The order of identification does not mean A is better than B , as the designations are only for identification purposes. For example: $\mathrm{A}_{12} \mathrm{~T}$ and $\mathrm{A}_{10} \mathrm{~T}$ imply company $\mathrm{A}$, twelve millimeter diameter for tensile test and company A, ten millimeter diameter for tension test respectively and so on. In each diameter, ten specimens were tested for complete test. The value presented in this paper is an average of ten (10) for each test ( be in bend or elongation).

\subsection{Samples Preparation}

Ten specimens were tested for each diameter as earlier stated. Each specimen consists of a length of 500 millimeters. Each specimen diameter is measured in three places and the average is obtained as the diameter. Then each specimen was subjected to tension in accordance with the BS4449:1997 provisions, and after fracture, the yield and ultimate strengths as well as characteristic strength were calculated. The results of the tensile tests are presented in tables 1 and 2 below:

\subsection{Tensile Tests} table 1 below:

The results of the tensile tests indicating the yield stress, ultimate stress and their ratios are as shown in

Table 1.0: Yield Stress, Ultimate Stress, Ultimate /Yield Ratio and Elongation.

\begin{tabular}{|c|c|c|c|c|c|}
\hline S/No & Mark & $\begin{array}{l}\text { Yield } \\
\left(\mathbf{N} / \mathbf{m m}^{2}\right)\end{array}$ Stress & $\begin{array}{ll}\text { Ultimate } \\
\left(\mathrm{N} / \mathbf{m m}^{2}\right)\end{array}$ Stress & $\begin{array}{l}\text { Ultimate/Yield, } \\
\text { Ratio }\end{array}$ & $\begin{array}{l}\text { Elongation } \\
(\%)\end{array}$ \\
\hline 01 & $\mathrm{~A}_{12} \mathrm{~T}$ & 357.43 & 529.83 & 1.55 & 16.50 \\
\hline 02 & $\mathrm{~A}_{10} \mathrm{~T}$ & 410.40 & 656.60 & 1.60 & 13.90 \\
\hline 03 & $\mathrm{~B}_{10} \mathrm{~T}$ & 390.30 & 536.60 & 1.46 & 19.60 \\
\hline 04 & $\mathrm{~B}_{8} \mathrm{~T}$ & 368.24 & 483.31 & 1.31 & 23.82 \\
\hline 05 & $\mathrm{C}_{16} \mathrm{~T}$ & 503.41 & 918.82 & 1.84 & 8.33 \\
\hline 06 & $\mathrm{C}_{10} \mathrm{~T}$ & 364.58 & 531.10 & 1.52 & 19.93 \\
\hline 07 & $\mathrm{C}_{8} \mathrm{~T}$ & 405.13 & 549.32 & 1.35 & 21.17 \\
\hline 08 & $\mathrm{D}_{8} \mathrm{~T}$ & 463.08 & 621.50 & 1.39 & 1.67 \\
\hline 09 & $\mathrm{E}_{25} \mathrm{~T}$ & 367.05 & 576.12 & 1.64 & 19.83 \\
\hline 10 & $\mathrm{E}_{20} \mathrm{~T}$ & 318.95 & 449.38 & 1.43 & 24.27 \\
\hline 11 & $\mathrm{~F}_{12} \mathrm{~T}$ & 415.60 & 633.21 & 1.52 & 19.07 \\
\hline 12 & $\mathrm{G}_{12} \mathrm{~T}$ & 419.56 & 715.25 & 1.69 & 14.83 \\
\hline 13 & $\mathrm{H}_{16} \mathrm{~T}$ & 499.26 & 599.75 & 1.22 & 14.53 \\
\hline 14 & $\mathrm{I}_{12} \mathrm{~T}$ & 369.11 & 612.53 & 1.69 & 14.50 \\
\hline 15 & $\mathrm{~J}_{8} \mathrm{~T}$ & 572.81 & 693.40 & 1.21 & 2.67 \\
\hline 16 & $\mathrm{~K}_{10} \mathrm{~T}$ & 574.38 & 660.17 & 1.22 & 10.07 \\
\hline 17 & $\mathrm{~L}_{12} \mathrm{~T}$ & 505.18 & 599.62 & 1.21 & 14.93 \\
\hline 18 & $\mathrm{M}_{10} \mathrm{~T}$ & 563.70 & 642.94 & 1.08 & 11.77 \\
\hline 19 & $\mathrm{~N}_{16} \mathrm{~T}$ & 551.72 & 645.41 & 1.26 & 13.90 \\
\hline
\end{tabular}

\subsection{Characteristic Strength}

Following the yield load, the yield stress and characteristic strengths were calculated using the code and the measured diameter were included as shown in table two (2) Below:

Table 2 : Characteristic Strength Tests Results with Corresponding Diameters.

\begin{tabular}{|l|l|l|l|}
\hline S/No & Mark & Measured Diameter $(\mathbf{m m})$ & Characteristic Strength $\left(\mathbf{N} / \mathbf{m m}^{2}\right)$ \\
\hline 01 & $\mathrm{~A}_{12} \mathrm{~T}$ & 11.88 & 350.00 \\
\hline 02 & $\mathrm{~A}_{10} \mathrm{~T}$ & 9.65 & 410.00 \\
\hline 03 & $\mathrm{~B}_{10} \mathrm{~T}$ & 9.56 & 390.00 \\
\hline 04 & $\mathrm{~B}_{8} \mathrm{~T}$ & 7.44 & 368.00 \\
\hline
\end{tabular}


Tensile Tests on Reinforcing Steel Bars in The Nigerian Construction Industry

\begin{tabular}{|l|l|l|l|}
\hline 05 & $\mathrm{C}_{16} \mathrm{~T}$ & 15.82 & 482.00 \\
\hline 06 & $\mathrm{C}_{10} \mathrm{~T}$ & 9.55 & 357.00 \\
\hline 07 & $\mathrm{C}_{8} \mathrm{~T}$ & 7.46 & 387.00 \\
\hline 08 & $\mathrm{D}_{8} \mathrm{~T}^{*}$ & 7.23 & 463.00 \\
\hline 09 & $\mathrm{E}_{25} \mathrm{~T}$ & 24.56 & 363.00 \\
\hline 10 & $\mathrm{E}_{20} \mathrm{~T}$ & 19.57 & 317.00 \\
\hline 11 & $\mathrm{~F}_{12} \mathrm{~T}$ & 11.4 & 334.00 \\
\hline 12 & $\mathrm{G}_{12} \mathrm{~T}$ & 11.48 & 408.00 \\
\hline 13 & $\mathrm{H}_{16} \mathrm{~T}$ & 15.52 & 493.00 \\
\hline 14 & $\mathrm{I}_{12} \mathrm{~T}$ & 11.40 & 369.00 \\
\hline 15 & $\mathrm{~J}_{8} \mathrm{~T}^{*}$ & 6.50 & 573.00 \\
\hline 16 & $\mathrm{~K}_{10} \mathrm{~T}^{*}$ & 9.36 & 549.00 \\
\hline 17 & $\mathrm{~L}_{12} \mathrm{~T}^{*}$ & 11.82 & 500.00 \\
\hline 18 & $\mathrm{M}_{10} \mathrm{~T}^{*}$ & 9.23 & 547.00 \\
\hline 19 & $\mathrm{~N}_{16} \mathrm{~T}^{*}$ & 15.60 & 545.00 \\
\hline
\end{tabular}

\section{Analyses And Discussion Of Results:}

The test results were analysed and discussed to arrive at a reasonable conclusion. It is important to note that each diameter comprises ten (10) specimens and average values were used throughout.

\subsection{Characteristic Strength}

The characteristic strengths computed from the yield strengths shown in table 2 Above Code Value and Below Code Value are compared with the code requirements, and are presented in table 3 Below:

Table 3.0: Comparison of Characteristic Strengths with Code Values.

\begin{tabular}{|l|l|l|l|l|}
\hline S/No & Mark & $\begin{array}{l}\text { Characteristic } \\
\text { Strength }\left(\mathbf{N} / \mathbf{m m}^{2}\right)\end{array}$ & BS4449(1997) Min. Provisions & Remarks \\
\hline $\mathbf{1}$ & $\mathrm{A}_{12} \mathrm{~T}$ & 350.00 & 460.00 & Below Code Value \\
\hline $\mathbf{2}$ & $\mathrm{A}_{10} \mathrm{~T}$ & 410.00 & 460.00 & Below Code Value \\
\hline $\mathbf{3}$ & $\mathrm{B}_{10} \mathrm{~T}$ & 390.00 & 460.00 & Below Code Value \\
\hline $\mathbf{4}$ & $\mathrm{B}_{8} \mathrm{~T}$ & 368.00 & 460.00 & Below Code Value \\
\hline $\mathbf{5}$ & $\mathrm{C}_{16} \mathrm{~T}$ & 482.00 & 460.00 & Above Code Value \\
\hline $\mathbf{6}$ & $\mathrm{C}_{10} \mathrm{~T}$ & 357.00 & 460.00 & Below Code Value \\
\hline $\mathbf{7}$ & $\mathrm{C}_{8} \mathrm{~T}$ & 387.00 & 460.00 & Below Code Value \\
\hline $\mathbf{8}$ & $\mathrm{D}_{8} \mathrm{~T}^{*}$ & 463.00 & 460.00 & Above Code Value \\
\hline $\mathbf{9}$ & $\mathrm{E}_{25} \mathrm{~T}$ & 363.00 & 460.00 & Below Code Value \\
\hline $\mathbf{1 0}$ & $\mathrm{E}_{20} \mathrm{~T}$ & 317.00 & 460.00 & Below Code Value \\
\hline $\mathbf{1 1}$ & $\mathrm{F}_{12} \mathrm{~T}$ & 334.00 & 460.00 & Below Code Value \\
\hline $\mathbf{1 2}$ & $\mathrm{G}_{12} \mathrm{~T}$ & 408.00 & 460.00 & Below Code Value \\
\hline $\mathbf{1 3}$ & $\mathrm{H}_{16} \mathrm{~T}$ & 493.00 & 460.00 & Above Code Value \\
\hline $\mathbf{1 4}$ & $\mathrm{I}_{12} \mathrm{~T}$ & 369.00 & 460.00 & Below Code Value \\
\hline $\mathbf{1 5}$ & $\mathrm{J}_{8} \mathrm{~T}^{*}$ & 573.00 & 460.00 & Above Code Value \\
\hline $\mathbf{1 6}$ & $\mathrm{K}_{10} \mathrm{~T}^{*}$ & 549.00 & 460.00 & Above Code Value \\
\hline $\mathbf{1 7}$ & $\mathrm{L}_{12} \mathrm{~T}^{*}$ & 500.00 & 460.00 & Above Code Value \\
\hline $\mathbf{1 8}$ & $\mathrm{M}_{10} \mathrm{~T}^{*}$ & 547.00 & 460.00 & Above Code Value \\
\hline $\mathbf{1 9}$ & $\mathrm{N}_{16} \mathrm{~T}^{*}$ & 545.00 & 460.00 & Above Code Value \\
\hline
\end{tabular}

* Implies foreign bars

Observing carefully, eleven out of nineteen samples $(58 \%<60 \%)$ fall below code value as the characteristic strength value as specified by the code $(460 \mathrm{~N} / \mathrm{mm} 2)$. This is not good enough as these values are usually used for design value ( $0.87 \mathrm{fy})$, where fy is the characteristic strength.

\subsection{Ultimate to Yield Strength Ratio}

Table 4 below shows the ratio of the ultimate to yield strength. It can be seen that the ultimate to yield strength ratio values in respect of all the nineteen samples are above the minimum code provisions which is 1.15 . The values obtained for the fourteen local samples are relatively higher than those obtained for the foreign bar samples, which are very close to the minimum. 
Tensile Tests on Reinforcing Steel Bars in The Nigerian Construction Industry

Table 4: Ultimate to Yield Strength Ratio Values for The Nineteen Samples

* Implies foreign bars

\begin{tabular}{|l|l|l|l|l|}
\hline S/N & Mark & $\begin{array}{l}\text { Ultimate/Yield } \\
\text { Strength Ratio }\end{array}$ & BS4449/ 1997 Min Provisions & Remarks \\
\hline $\mathbf{1}$ & $\mathrm{A}_{12} \mathrm{~T}$ & 1.55 & 1.15 & Above Code Value \\
\hline $\mathbf{2}$ & $\mathrm{A}_{10} \mathrm{~T}$ & 1.60 & 1.15 & Above Code Value \\
\hline $\mathbf{3}$ & $\mathrm{B}_{10} \mathrm{~T}$ & 1.46 & 1.15 & Above Code Value \\
\hline $\mathbf{4}$ & $\mathrm{B}_{8} \mathrm{~T}$ & 1.31 & 1.15 & Above Code Value \\
\hline $\mathbf{5}$ & $\mathrm{C}_{16} \mathrm{~T}$ & 1.84 & 1.15 & Above Code Value \\
\hline $\mathbf{6}$ & $\mathrm{C}_{10} \mathrm{~T}$ & 1.52 & 1.15 & Above Code Value \\
\hline $\mathbf{7}$ & $\mathrm{C}_{8} \mathrm{~T}$ & 1.35 & 1.15 & Above Code Value \\
\hline $\mathbf{8}$ & $\mathrm{D}_{8} \mathrm{~T}^{*}$ & 1.39 & 1.15 & Above Code Value \\
\hline $\mathbf{9}$ & $\mathrm{E}_{25} \mathrm{~T}$ & 1.64 & 1.15 & Above Code Value \\
\hline $\mathbf{1 0}$ & $\mathrm{E}_{20} \mathrm{~T}$ & 1.43 & 1.15 & Above Code Value \\
\hline $\mathbf{1 1}$ & $\mathrm{F}_{12} \mathrm{~T}$ & 1.52 & 1.15 & Above Code Value \\
\hline $\mathbf{1 2}$ & $\mathrm{G}_{12} \mathrm{~T}$ & 1.69 & 1.15 & Above Code Value \\
\hline $\mathbf{1 3}$ & $\mathrm{H}_{16} \mathrm{~T}$ & 1.22 & 1.15 & Above Code Value \\
\hline $\mathbf{1 4}$ & $\mathrm{I}_{12} \mathrm{~T}$ & 1.69 & 1.15 & Above Code Value \\
\hline $\mathbf{1 5}$ & $\mathrm{J}_{8} \mathrm{~T}^{*}$ & 1.21 & 1.15 & Above Code Value \\
\hline $\mathbf{1 6}$ & $\mathrm{K}_{10} \mathrm{~T}^{*}$ & 1.22 & 1.15 & Above Code Value \\
\hline $\mathbf{1 7}$ & $\mathrm{L}_{12} \mathrm{~T}^{*}$ & 1.21 & 1.15 & Above Code Value \\
\hline $\mathbf{1 8}$ & $\mathrm{M}_{10} \mathrm{~T}^{*}$ & 1.08 & 1.15 & Below Code Value \\
\hline $\mathbf{1 9}$ & $\mathrm{N}_{16} \mathrm{~T}^{*}$ & 1.26 & 1.15 & Above Code Value \\
\hline & & & & \\
\hline
\end{tabular}

It can be observed that bars samples $\mathrm{M}_{10} \mathrm{~T}$ which is a foreign sample is Below Code Value minimum requirement. This could be as a result of cooling process which is a manufacturing fault within the production line. However, when the ratio is high, it is not good either. It implies high carbon content which may lack ductility.

\subsection{Percentage Elongation}

Table 5 shows percentage elongation for the nine samples collected. From the table 5 below, it can be seen that most of the local bar samples met the minimum code requirements on elongation, while most of the foreign bars did not meet the minimum codes requirements. Serial numbers 5, 8, 15, 16 and 18 failed to reach the value of 14 percent and on observing carefully serial numbers 5, 6 and 7 are of the same company, but sample no. 5 failed to satisfy the elongation requirement. Secondly, samples nos. 15 to 19 and also no. 8 are foreign companies and only the sample with serial number 17 passed.

Table 5: Percentage Elongation Values For The Nineteen Samples

\begin{tabular}{|l|l|l|l|l|}
\hline S/No & Mark & Elongation $(\%)$ & Min. BS4449/1997 Provisions & Remarks \\
\hline $\mathbf{1}$ & $\mathrm{A}_{12} \mathrm{~T}$ & 16.50 & 14.00 & Above Code Value \\
\hline $\mathbf{2}$ & $\mathrm{A}_{10} \mathrm{~T}$ & 13.90 & 14.00 & Below Code Value \\
\hline $\mathbf{3}$ & $\mathrm{B}_{10} \mathrm{~T}$ & 19.60 & 14.00 & Above Code Value \\
\hline $\mathbf{4}$ & $\mathrm{B}_{8} \mathrm{~T}$ & 23.82 & 14.00 & Above Code Value \\
\hline $\mathbf{5}$ & $\mathrm{C}_{16} \mathrm{~T}$ & 8.33 & 14.00 & Below Code Value \\
\hline $\mathbf{6}$ & $\mathrm{C}_{10} \mathrm{~T}$ & 19.93 & 14.00 & Above Code Value \\
\hline $\mathbf{7}$ & $\mathrm{C}_{8} \mathrm{~T}$ & 21.17 & 14.00 & Above Code Value \\
\hline $\mathbf{8}$ & $\mathrm{D}_{8} \mathrm{~T}^{*}$ & 1.67 & 14.00 & Below Code Value \\
\hline $\mathbf{9}$ & $\mathrm{E}_{25} \mathrm{~T}$ & 19.83 & 14.00 & Above Code Value \\
\hline $\mathbf{1 0}$ & $\mathrm{E}_{20} \mathrm{~T}$ & 24.27 & 14.00 & Above Code Value \\
\hline $\mathbf{1 1}$ & $\mathrm{F}_{12} \mathrm{~T}$ & 19.07 & 14.00 & Above Code Value \\
\hline $\mathbf{1 2}$ & $\mathrm{G}_{12} \mathrm{~T}$ & 14.83 & 14.00 & Above Code Value \\
\hline $\mathbf{1 3}$ & $\mathrm{H}_{16} \mathrm{~T}$ & 14.53 & 14.00 & Above Code Value \\
\hline $\mathbf{1 4}$ & $\mathrm{I}_{12} \mathrm{~T}$ & 14.50 & 14.00 & Above Code Value \\
\hline $\mathbf{1 5}$ & $\mathrm{J}_{8} \mathrm{~T}^{*}$ & 2.67 & 14.00 & Below Code Value \\
\hline $\mathbf{1 6}$ & $\mathrm{K}_{10} \mathrm{~T}^{*}$ & 10.07 & 14.00 & Below Code Value \\
\hline $\mathbf{1 7}$ & $\mathrm{L}_{12} \mathrm{~T}^{*}$ & 14.93 & 14.00 & Above Code Value \\
\hline $\mathbf{1 8}$ & $\mathrm{M}_{10} \mathrm{~T}^{*}$ & 11.77 & 14.00 & Below Code Value \\
\hline $\mathbf{1 9}$ & $\mathrm{N}_{16} \mathrm{~T}^{*}$ & 13.90 & 14.00 & Below Code Value \\
\hline
\end{tabular}


These samples that failed in elongation should not be used in reinforcement as they will not give warning prior to failure due to low ductility. This lack of ductility usually leads to sudden collapse without warning.

\subsection{Other Some Measured Parameters:}

Some measured parameters during the tension test were selected and compared to design strength. The minimum design strength for reinforcement is $0.87 \mathrm{fy}$, where fy is the characteristic strength. In table 4.6 below , the characteristic strengths are compared with the design strength which is $400 \mathrm{~N} / \mathrm{mm}^{2}$, when the minimum characteristic strength is $460 \mathrm{~N} / \mathrm{mm}^{2}$ as specified by the code.

Table 6 : Comparison of Characteristic Strength with Design Strength.

\begin{tabular}{|c|c|c|c|c|c|c|}
\hline S/No & Mark & $\begin{array}{l}\text { Characteristic } \\
\text { Strength } \\
\left(\mathrm{N} / \mathbf{m m}^{2}\right) \\
\end{array}$ & $\begin{array}{l}\text { Measured Design } \\
\text { Strength }(\mathrm{N} / \mathbf{m m} 2)\end{array}$ & $\begin{array}{l}\text { Design Strength } \\
\left(0.87 \text { fy- } \mathrm{N} / \mathrm{mm}^{2}\right)\end{array}$ & $\begin{array}{l}\text { Difference } \\
(\%)\end{array}$ & Remarks \\
\hline 01 & $\mathrm{~A}_{12} \mathrm{~T}$ & 350.00 & 305.00 & 400.00 & -24.00 & Unsatisfactory \\
\hline 02 & $\mathrm{~A}_{10} \mathrm{~T}$ & 410.00 & 357.00 & 400.00 & -11.00 & Unsatisfactory \\
\hline 03 & $\mathrm{~B}_{10} \mathrm{~T}$ & 390.00 & 339.00 & 400.00 & -15.00 & Unsatisfactory \\
\hline 04 & $\mathrm{~B}_{8} \mathrm{~T}$ & 368.00 & 320.00 & 400.00 & -20.00 & Unsatisfactory \\
\hline 05 & $\mathrm{C}_{16} \mathrm{~T}$ & 482.00 & 419.00 & 400.00 & +5.00 & Satisfactory \\
\hline 06 & $\mathrm{C}_{10} \mathrm{~T}$ & 357.00 & 311.00 & 400.00 & -22.00 & Unsatisfactory \\
\hline 07 & $\mathrm{C}_{8} \mathrm{~T}$ & 387.00 & 337.00 & 400.00 & -16.00 & Unsatisfactory \\
\hline 08 & $\mathrm{D}_{8} \mathrm{~T}^{*}$ & 463.00 & 402.00 & 400.00 & +1.00 & Satisfactory \\
\hline 09 & $\mathrm{E}_{25} \mathrm{~T}$ & 363.00 & $316 . .00$ & 400.00 & -21.00 & Unsatisfactory \\
\hline 10 & $\mathrm{E}_{20} \mathrm{~T}$ & 317.00 & 276.00 & 400.00 & -31.00 & Unsatisfactory \\
\hline 11 & $\mathrm{~F}_{12} \mathrm{~T}$ & 334.00 & 291.00 & 400.00 & -27.00 & Unsatisfactory \\
\hline 12 & $\mathrm{G}_{12} \mathrm{~T}$ & 408.00 & 355.00 & 400.00 & -11.00 & Unsatisfactory \\
\hline 13 & $\mathrm{H}_{16} \mathrm{~T}$ & 493.00 & 429.00 & 400.00 & +7.00 & Satisfactory \\
\hline 14 & $\mathrm{I}_{12} \mathrm{~T}$ & 369.00 & 321.00 & 400.00 & -20.00 & Unsatisfactory \\
\hline 15 & $\mathrm{~J}_{8} \mathrm{~T}^{*}$ & 573.00 & 499.00 & 400.00 & +25.00 & Satisfactory \\
\hline 16 & $\mathrm{~K}_{10} \mathrm{~T}^{*}$ & 549.00 & 78.00 & 400.00 & +20.00 & Satisfactory \\
\hline 17 & $\mathrm{~L}_{12} \mathrm{~T}^{*}$ & 500.00 & 435.00 & 400.00 & +9.00 & Satisfactory \\
\hline 18 & $\mathrm{M}_{10} \mathrm{~T}^{*}$ & 547.00 & 476.00 & 400.00 & +19.00 & Satisfactory \\
\hline 19 & $\mathrm{~N}_{16} \mathrm{~T}^{*}$ & 545.00 & 474.00 & 400.00 & +19.00 & Satisfactory \\
\hline
\end{tabular}

From table 6, it can be observed that eleven (11) samples which are mostly local steel reinforcement out of fourteen local ones 78.6 percent of local reinforcement failed to meet up with the minimum design strength of $400 \mathrm{~N} / \mathrm{mm}^{2}$. That is to say seventy eight point six percent $(78.6 \%)$ of the local samples failed to meet up with the minimum design strength. This is very dangerous. The summary of the various parameter values for the different diameters tested as shown in table 7 below:

Table 7a: Parameter Summary for Tensile Tests (Companies A To E):

\begin{tabular}{|l|l|l|l|l|l|l|l|l|l|l|l|}
\hline S/No. & $\begin{array}{l}\text { SAMPLE } \\
\text { PARAMETER }\end{array}$ & $\mathbf{A}_{\mathbf{1 2}} \mathbf{T}$ & $\mathbf{A}_{\mathbf{1 0}} \mathbf{T}$ & $\mathbf{B}_{\mathbf{1 0}} \mathbf{T}$ & $\mathbf{B}_{\mathbf{8}} \mathbf{T}$ & $\mathbf{C}_{\mathbf{1 6}} \mathbf{T}$ & $\mathbf{C}_{\mathbf{1 0}} \mathbf{T}$ & $\mathbf{C}_{\mathbf{8}} \mathbf{T}$ & $\mathbf{D}_{\mathbf{8}} \mathbf{T}$ & $\mathbf{E}_{\mathbf{2 5}} \mathbf{T}$ & $\mathbf{E}_{\mathbf{2 0}} \mathbf{T}$ \\
\hline 01 & Diameter(mm) & 12 & 10 & 10 & 8 & 16 & 10 & 8 & 8 & 25 & 20 \\
\hline 02 & $\begin{array}{l}\text { Characteristic } \\
\left.\text { Strength(N/mm }{ }^{2}\right)\end{array}$ & 350. & 410.0 & 390. & 368.2 & 482. & 357. & 387. & 463. & 363.0 & 317. \\
& 0 & 3 & & 0 & 0 & 0 & 0 & & 0 \\
\hline 03 & $\begin{array}{l}\text { Standard } \\
\text { Deviation }\end{array}$ & 4.65 & 0.0 & 0.0 & 0.0 & 13.0 & 4.4 & 11.1 & 0.0 & 2.4 & 1.0 \\
\hline
\end{tabular}

Table 7b: Parameter Summary for Tensile Tests(Companies F To N):

\begin{tabular}{|c|c|c|c|c|c|c|c|c|c|c|}
\hline $\begin{array}{l}\text { S/N } \\
\text { o. }\end{array}$ & $\begin{array}{l}\text { SAMPLE } \\
\text { PARAMETER }\end{array}$ & $\mathrm{F}_{12} \mathrm{~T}$ & $\mathbf{G}_{8} T$ & $\mathrm{H}_{12} \mathrm{~T}$ & $\mathrm{I}_{16} \mathrm{~T}$ & $\mathbf{J}_{12} \mathbf{T}$ & $K_{10} T$ & $\mathbf{L}_{12} \mathrm{~T}$ & $M_{10} T$ & $\mathrm{~N}_{16} \mathrm{~T}$ \\
\hline 01 & Diameter(mm) & 12 & 8 & 12 & 16 & 12 & 10 & 12 & 10 & 16 \\
\hline 02 & $\begin{array}{l}\text { Characteristic } \\
\text { Strength }\left(\mathrm{N} / \mathrm{mm}^{2}\right)\end{array}$ & 334.0 & 573.0 & 408.0 & 493.0 & 369.0 & 549.0 & 500.0 & 547.0 & 546.0 \\
\hline 03 & Standard Deviation & 49.6 & 0.0 & 6.8 & 3.7 & 0.0 & 15.71 & 2.9 & 10.1 & 3.7 \\
\hline
\end{tabular}

The value of standard deviation will determine the skills of the people that are under employment. The small the value indicates high skilled personnel were employed. Values below or equal to five are an indicative of highly skilled personnel and Above Code Value five indicates employment of low skilled men which lead to bad products, etc. About five samples from company C, F, H, K and M have values Above Code Value five. A 
balance must be maintained for effective administration and good output .The five companies mentioned above employed unskilled labour leading to poor product production.

\subsection{Cross - Checking Samples with Parameters Tested}

From table 8 below, tensile test parameters are cross checked with each diameter samples and see if each parameter sample passed all the parameters. Such parameters are Characteristics Strength and Ultimate to Yield strength ratio. The remark column indicates how each diameter sample performed. The keys are given at the end of the table.

The characteristic strength was calculated from the equation $f y=f a v-1.64 \mathrm{SD}$ where; fy is the characteristic strength, $\mathrm{f}$ av is the average yield stress and $\mathrm{SD}$ is the standard deviation.

TABLE 3.6: TESTING OF SOME SELECTED BEND AND TENSILE TEST PARAMETERS.

\begin{tabular}{|l|l|l|l|l|l|}
\hline S/No & Mark & $\begin{array}{l}\text { Characteristic Strength } \\
\left(\mathbf{N} / \mathbf{m m}^{2}\right)\end{array}$ & Elongation $(\boldsymbol{\%})$ & Ultimate : Yield Str. Ratio & Remarks \\
\hline $\mathbf{1}$ & $\mathrm{A}_{12} \mathrm{~T}$ & $\times$ & $\sqrt{ }$ & $\sqrt{ }$ & Partially Complied \\
\hline $\mathbf{2}$ & $\mathrm{A}_{10} \mathrm{~T}$ & $\times$ & $\times$ & $\sqrt{ }$ & Partially Complied \\
\hline $\mathbf{3}$ & $\mathrm{B}_{10} \mathrm{~T}$ & $\times$ & $\sqrt{ }$ & $\sqrt{ }$ & Partially Complied \\
\hline $\mathbf{4}$ & $\mathrm{B}_{8} \mathrm{~T}$ & $\times$ & $\sqrt{ }$ & $\sqrt{ }$ & Partially Complied \\
\hline $\mathbf{5}$ & $\mathrm{C}_{16} \mathrm{~T}$ & $\sqrt{ }$ & $\times$ & $\sqrt{ }$ & Partially Complied \\
\hline $\mathbf{6}$ & $\mathrm{C}_{10} \mathrm{~T}$ & $\times$ & $\sqrt{ }$ & $\sqrt{ }$ & Partially Complied \\
\hline $\mathbf{7}$ & $\mathrm{C}_{8} \mathrm{~T}$ & $\times$ & $\sqrt{ }$ & $\sqrt{ }$ & Partially Complied \\
\hline $\mathbf{8}$ & $\mathrm{D}_{8} \mathrm{~T}^{*}$ & $\sqrt{ }$ & $\times$ & $\sqrt{ }$ & Partially Complied \\
\hline $\mathbf{9}$ & $\mathrm{E}_{25} \mathrm{~T}$ & $\times$ & $\sqrt{ }$ & $\sqrt{ }$ & Partially Complied \\
\hline $\mathbf{1 0}$ & $\mathrm{E}_{20} \mathrm{~T}$ & $\times$ & $\sqrt{ }$ & $\sqrt{ }$ & Partially Complied \\
\hline $\mathbf{1 1}$ & $\mathrm{F}_{12} \mathrm{~T}$ & $\times$ & $\sqrt{ }$ & $\sqrt{ }$ & Partially Complied \\
\hline $\mathbf{1 2}$ & $\mathrm{G}_{12} \mathrm{~T}$ & $\times$ & $\sqrt{ }$ & $\sqrt{ }$ & Partially Complied \\
\hline $\mathbf{1 3}$ & $\mathrm{H}_{16} \mathrm{~T}$ & $\sqrt{ }$ & $\sqrt{ }$ & $\sqrt{ }$ & Partially Complied \\
\hline $\mathbf{1 4}$ & $\mathrm{I}_{12} \mathrm{~T}$ & $\times$ & $\sqrt{ }$ & $\sqrt{ }$ & Partially Complied \\
\hline $\mathbf{1 5}$ & $\mathrm{J}_{8} \mathrm{~T}^{*}$ & $\sqrt{ }$ & $\times$ & $\times$ & Partially Complied \\
\hline $\mathbf{1 6}$ & $\mathrm{K}_{10} \mathrm{~T}^{*}$ & $\sqrt{ }$ & $\times$ & $\sqrt{ }$ & Partially Complied \\
\hline $\mathbf{1 7}$ & $\mathrm{L}_{12} \mathrm{~T}^{*}$ & $\sqrt{ }$ & $\sqrt{ }$ & $\sqrt{ }$ & Partially Complied \\
\hline $\mathbf{1 8}$ & $\mathrm{M}_{10} \mathrm{~T}^{*}$ & $\sqrt{ }$ & $\times$ & $\times$ & Partially Complied \\
\hline $\mathbf{1 9}$ & $\mathrm{N}_{16} \mathrm{~T}^{*}$ & $\sqrt{ }$ & $\times$ & $\sqrt{ }$ & Partially Complied \\
\hline
\end{tabular}

\section{Legend: $\quad \sqrt{ } \Rightarrow$ Satisfactory ; $\quad \mathbf{X} \Rightarrow$ Unsatisfactory}

When all parameters measured as indicated above are compared with code specified, it is observed that it all indicated for the entire samples that they are not totally compliant with the code requirement.

\section{Conclusion}

Based on the tensile test conducted and the analyses/ observations carried out the following conclusions were made.

1. The characteristic strength values for most of the locally produced bar samples are low compared to the BS4449:1969,1995\& 1997 standards for high tensile steel which is $460 \mathrm{~N} / \mathrm{mm}^{2}$ minimum value. Sixty percent of the sample fall Below Code Value .

2. The characteristic strength values in respect of the local bars suggest similarities with characteristics strength of mild steel. This implies the products are actually mild steel and are retread and openly sold as high tensile steel in the market.

3. Most of the locally produced bars have recorded satisfactory percentage elongation with corresponding unsatisfactory characteristic strength values and vice versa in the case of foreign bars.

4. Most of the reinforcement bar samples complied with the minimum ultimate to yield strength ratio as specified by BS 4449: 1969 and 1997 code provisions.

5. Seventy One percent $(71 \%)$ of the samples design strength value failed below the code specified design values. 
6. Five of the fourteen companies (thirty six percent) ie $36 \%$ of the companies where samples were collected used unskilled labour in their factory production as such, companies are hardly checked by Government Agency.

7. From the characteristic and design strengths, most of the high tensile steel in market in the Nigerian Construction Industry are retreaded mild steel as high tensile steel. This should be carefully checked.

8. A constant check and re evaluation by Government agency within the industry is recommended and cannot be over emphasized.

\section{Acknowlegement}

The kind permission of Professor (Mrs) Gambo Laraba Abdullahi, Director General and Chief Executive Officer, National Board for Technology Incubation, Abuja - Nigeria, former Honourable Minister for Women Affairs - Federal Republic of Nigeria, Recipient of the Nigerian National Honours, etc., to undertake the successful conduct of the experiments is highly appreciated. Her effort and good administrative skills are also acknowledged.

\section{References}

[1] Arum, C. (2008): "Verification of Properties of Concrete Reinforcement Bars:- Nigerian Case Study" - Published by : A Journal of Indoor and Built Environment by SAGE Publications. http:ibe.sagepub.com PP.370-376

[2] American Society for Testing of Materials (2003): AASHTO No. M 31 "Standard Specifications for Deformed \& Plain Billet Steel Bars for Concrete Reinforcement": American Association of Highway and Transport Officials - A615/A615M- 03a

[3] American Society for Testing of Materials: (2007) "Standard Test Methods and Definitions for Mechanical Testing of Steel Products": ASTM A37-07a

[4] American Society for Testing of Materials: (1988) "Standard Specifications for Structural Steel", ASTM - A38/A38M, 25 ${ }^{\text {th }}$ edition, pp. 105

[5] American Society for Testing of Materials: (1988) "Standard Specifications for Structural Steel", ASTM - A38/A38M, 25 ${ }^{\text {th }}$ edition, pp.562

[6] British Standards Institutions. BS 4449(1997) -“Carbon Steel Bars for the Reinforcement of concrete” London. pp.1-17

[7] Charles K.K. and Mark A (2002) "Strength \& Ductility Characteristics of Reinforcing Steel Bar milled from Scrap Metals"Materials and Design 23 pp.537-545

[8] Certification Authority for Reinforcement Steels (1984-1995) (UK CARES Vol. 1 - 10).

[9] FIROZE, P.E. Engr. "Attributes of Ductile Reinforcing Steel" - PDF Extracts from internet - m.firoze@ bsm.com . pp. 18-20

[10] Jibrin, M.U. (2012): "Characterisation of Reinforcing Bars in the Nigerian Construction Industry" A Ph.D Thesis in Civil Engineering , Ahmadu Bello University, Zaria - Nigeria. PP. 44 - 94

[11] Kareem, B. (2009): Tensile \& Chemical Analyses of Selected Steel Bars. AU J.T. 13(1): 29 - 33.

[12] Nigerian Industrial Standard - NIS- 157 : 1982 Specification for Bending Dimensions and Scheduling of Bars For The Reinforcement of Concrete PP. 4/2.

[13] Nigerian Industrial Standard (1973) - NIS-56 :"Specification for Mild Steel Wires for Iron and Steel in the Steel Making Industry" PP. 5 - 23.

[14] Nigerian Industrial Standard (1992) - NIS-117: Specification for Steel Bars for Reinforcement of Concrete (Reviewed) Published by The Standards Organisation of Nigeria- PP. $3-15$

[15] Raw Materials Research and Development Council (2006), FMST- "Industrial Studies on Base Metal, Iron and Steel and Engineering Services Sector $\left(5^{\text {th }}\right.$ Update $)$. All pages.

[16] Sanmbo, B., David , E., Adeosun, S., and Olatunde, S.(2009): "Challenges of Producing Quality Construction Steel Bars in West Africa". Journal of Minerals and Materials Characterization and Engineering. Vol. 8, No. 4, pp 283-292.

[17] Shumacher, K and Sathaye, J. (1998) : India's Iron and Steel Industry : Lawrence Berkeley National Library, Beckley. PP. 4 \&5. 